work day or on way from work to home. This database is very reliable and gives a good outlook of happened injuries and accidents.

Posti is a big organisation where employees are working outside when delivering newspapers (early in the morning) and magazines and other post (in the daytime). Posti has their own database that includes information about injuries and other related data.

The information of slipping injuries from different sources and weather data are compared in this study.

Results The level of slipping injuries is clearly higher during winter time than during summer time. There seems to be quite visible correlation between temperature and number of slipping injuries, sometimes also snowfall seems to correlate quite well with the number of daily injuries. When temperature drops below zero degrees the number of slipping injuries use to raise.

Conclusions Slipping injuries are very common problem especially in countries located in places where ice and snow exist on winter time. There should be lot of potential available to decrease the number of slip injuries. This study presents the statistics of the slip injuries compared to weather. Also, suggestions are given how the awareness of the slipperiness could be improved.

\section{SPONSOR BEHAVIOUR AND IMPACTS IN PUBLIC SECTOR PROJECT MANAGEMENT}

Tarja Kantolahti. Ministry of Social Affairs and Health, Finland

10.1136/injuryprev-2016-042156.588

Background Project management literature does not describe how the sponsor behaves during project control in this specific occupational safety and health (OSH) public sector project context. Project management literature does not describe how the sponsor perceives the impacts of projects in this OSH context. It also seems that the literature does not recognise how the behaviour of the sponsor is related to sponsor perceptions of project impact. The objective of this study is to increase understanding of sponsor behaviour and impacts in public sector projects from the viewpoint of the sponsors themselves.

Methods This study is based on the qualitative method. Interviewees were selected on the basis of archive material. Interviews were organised for twenty sponsors, and the experiences of the sponsors were analysed on the basis of Grounded Theory.

Results The study indicated that sponsor behaviour was polymorphic. The main terms found for sponsor were bureaucrat, participator, expert and observer. The results indicated that the sponsor recognised many impact dimensions. Connexions were found between the behaviour of the sponsor and the impact dimensions that were determined from the perceptions of the sponsor. This study also suggests that there is a relationship between the behaviour of the sponsor and how the sponsor recognised the impact dimensions of the project.

Conclusions This study increases the understanding that other sponsors, ministries, researchers and project executors have regarding sponsor actions in the OSH field. It provides better possibilities for open discussion of sponsor activities in public sector projects. The study provides improved opportunities for continuing discussion about the impact of projects. According to the study, more research is needed on sponsor behaviour.

\section{PHYSICAL DEMANDS OF FIRE FIGHTING FOR SEAFARERS-AN EMERGING ASPECT OF OCCUPATIONAL SAFETY IN SEAFARING}

Susanna Visuri, Paivi Milunpalo, Harri Lindholm, Sirpa Lusa, Ari-Pekka Rauttola, Mia Pylkkonen. Finnish Institute of Occupational Health

\subsection{6/injuryprev-2016-042156.589}

Background All seafarers with designated emergency tasks must take part to a basic safety training including a course of basic fire fighting despite their physical fitness. Physical fitness of seafarers is often unsatisfactory, obesity and ageing impair it even further. There is not much information about the physical strain of the courses' exercises for seafarers. Therefore the aim of the study was to measure physical strain of seafarers during a fire fighting course.

Methods Fourteen male master mariner students aged 19-21 attended to a simulated smoke-diving drill with self-contained breathing apparatus (SCBA). Perceived exertion was assessed by Borg scale and energetic strain was assessed by estimating oxygen consumption indirectly with heart rate variability method. Students conducted two exercises in pairs with SCBA. In the first exercise, each pair walked through warm, smoke filled enclosed spaces. The second exercise started with a fire attack and continued by searching and rescuing a victim (a doll, weight $30 \mathrm{~kg}$ ).

Results The first exercise lasted on average 14 minutes. During the exercise, the highest heart rate (HR) level was on average $145(123-169) \mathrm{b} / \mathrm{min}$ and the maximum oxygen intake $\left(\mathrm{VO}_{2} \mathrm{max}\right) 34(25-42) \mathrm{ml} / \mathrm{min} / \mathrm{kg}$. The physical load was 7 (3-10) MET and perceived exertion on average was 11 (7-15). The second exercise lasted on average 12 minutes. The highest HR level was on average 167 (126-181) b/min and $\mathrm{VO}_{2}$ max was 40 (2749) $\mathrm{ml} / \mathrm{min} / \mathrm{kg}$. The physical load of exercise was 10 (6-12) MET and the perceived exertion on average was 13 (9-15).

Conclusions Seafarers' safe performance during basic fire fighting course requires aerobic fitness equivalent to extremely vigorous intensity activities (like running stairs up). The real-life smoke-diving duties on ship have been reported to be even more strainful. For the safety of seafarers, both promotion of physical fitness and regular training of emergency duties should be seen as an occupational safety issue.

\section{Trauma Care and Rehabilitation}

\section{Post Mon 1.18}

\section{QUALITATIVE EVALUATION OF TRAUMA DELAYS IN ROAD TRAFFIC INJURY PATIENTS IN MARINGÁ, BRAZIL}

${ }^{1,2}$ Anjni Patel, ${ }^{2,3}$ Joao Ricardo Vissoci, ${ }^{2}$ Catherine Staton. 'Emory University, Atlanta, GA; ${ }^{2}$ Duke University, Durham, NC; ${ }^{3}$ Faculdade Ingá, Maringá, Brazil

\subsection{6/injuryprev-2016-042156.590}

Background Road traffic injuries (RTIs) are the 8th leading cause of death worldwide, with $90 \%$ occurring in low- and middleincome countries (LMICs). In Brazil, more than 43,800 people are killed by RTI annually. There is limited research evaluating RTI transport delays to trauma centres in LMICs. The objective of this study is to determine specific causes of prehospital transport delays in RTI patients to trauma centres in Maringá, Brazil. Methods We qualitatively evaluated the regional public prehospital system, Serviços de Atendimento Móvel de Urgência (SAMU), 
with a catchment area of 500,000 people, one Advanced and 4 Basic Life Support teams. We used a qualitative approach and interviewed providers. Inclusion criteria were: healthcare professionals working with SAMU or the regional trauma hospital Metropolitano for at least 6 months. Interviews focused on primary causes and measures to reduce delays in care of RTI patients.

Results We interviewed 11 providers: 2 physicians and 2 nurses from Metropolitano and 7 SAMU employees (1 nurse, 3 physicians, 3 drivers). Primary causes of delays fell into the following categories: 1) lack of public education, 2) traffic, 3) insufficient personnel/ambulances, 4) bureaucracy, and 5) poor location of stations. Traffic was the most common response, with a total of 7 responses, including nearly all SAMU providers. Suggested measures to reduce delays were: 1) improving public education, 2) increasing personnel, 3) increasing ambulances, 4) proper extrication/need for rapid treatment, and 5) need for a centralised station to avoid traffic. The most common response was the need for public education, primarily teaching drivers about ambulance right-of-way.

Conclusions Most providers, particularly SAMU providers, believe traffic is the primary cause of delay in presentation of RTI patients to a tertiary care centre. Rapid economic growth and increased road traffic are primary factors leading to the overall increased rates of RTIs in LMICs. The same traffic causing RTIs may also be a significant cause of delay in the treatment these patients. Offered solutions to reduce delays focused mostly on public education and the need for increased resources. A public education campaign for driver education in response to RTIs could be an initial step towards reducing delays in the care of RTI patients.

\section{THE AGREEMENT OF TRIAGE RESULT BY REGISTERED NURSE AT DISPATCH CENTRE, ON SCENE AND EMERGENCY ROOM}

${ }^{1}$ Daoruang Kommuangpuk, ${ }^{2}$ Anuchar Sethasathien. ' Udonthani Hospital, Thailand; ${ }^{2}$ National Institute for Emergency Medicine, Thailand

\subsection{6/injuryprev-2016-042156.591}

Background The $8^{\text {th }}$ regional health area of Thailand, have 7 province and 88 hospitals. Three phase triage, first at dispatch centre second on scene and third in emergency room (ER) were done by difference registered nurses (RN). One patient will be 3 times triage by 3 difference $\mathrm{RN}$.

Methods This Quasi-experimental study focus on agreement of triage result by RN from 88 hospitals, compared between before and after triage training. RN was practically trained, triage competency development for modified Emergency Severity Index (ESI), on June 2014. The data was collected on May and July 2014. Analysed by kappa statistics and 95\% CI: of kappa. The percent agreement before and after training triage was compared by Chi-square test.

Results 3,325 patients were triaged, almost triage level were yellow and red. There were 24 Clinical-based categories of Emergency service. Top three of the Categories were Motor Vehicle Accident (22.20\%) Sick (17.75\%) Unconscious (8.30\%). Before training, the agreement between dispatch and scene was almost perfect with kappa 0.82 (95\% CI: $=0.78-0.87)$ the agreement between scene and ER was moderate with kappa 0.47 (95\% CI: $=0.42-0.52)$. After training, the agreement between dispatch and scene was almost perfect with kappa 0.85 (95\% CI: $=0.80-$
0.89) the agreement between scene and ER was moderate with kappa 0.57 (95\% CI: $=0.52-0.61)$. The percent agreement of dispatch and scene triage between before and after training were not different. But the percent agreement of scene and ER triage were significantly improved (P-value $=0.001$ ), with 0.05 level of significance.

Conclusions The agreement of scene and ER triage was moderate, since patients were received first aid and treatment in transit that did change for level of triage when they came to ER. But after training, this agreement was better than before. This study confirm that we can improve triage agreement for $\mathrm{RN}$ by triage training and we should improve the other first aid and treatment competency for RN.

\section{INJURED PATIENTS' INTERACTIONS WITH HEALTH PROVIDERS: IMPLICATIONS FOR ENHANCING TRAUMA CARE}

${ }^{1}$ Sandy Braaf, ${ }^{2}$ Shanthi Ameratunga, ${ }^{3}$ Nicola Christie, ${ }^{1}$ Peter Cameron, ${ }^{4}$ Ronan Lyons, ${ }^{5}$ James Harrison, ${ }^{1}$ Jennie Ponsford, ${ }^{1}$ Alex Collie, ${ }^{6}$ Mark Fitzgerald, ${ }^{7}$ Rodney Judson, ${ }^{8}$ Andrew Nunn, ${ }^{9}$ David Attwood, ${ }^{10}$ Helen Jowett, ${ }^{10}$ Warwick Teague, ${ }^{1}$ Belinda Gabbe. ${ }^{1}$ Monash University, Australia; ${ }^{2}$ University of Auckland, New Zealand; ${ }^{3}$ University College London, United Kingdom; ${ }^{4}$ Swansea University, United Kingdom; ; ${ }^{5}$ Flinders University, Australia; ${ }^{6}$ The Alfred, Australia; ${ }^{7}$ Royal Melbourne Hospital, Australia; ${ }^{8}$ Victorian Spinal Cord Service, Australia; ${ }^{9}$ Transport Accident Commission, Australia; ${ }^{10}$ Royal Children's Hospital, Australia

\subsection{6/injuryprev-2016-042156.592}

Background The path to recovery following major trauma can involve a long trajectory of complex health care needs and multiple interactions with health professions. We explored the perspectives of seriously injured patients regarding issues that arise during their interactions with health providers.

Methods This qualitative study, nested within a population-based longitudinal cohort study, involved semi-structured telephone interviews conducted three years following injury with 64 adult major trauma patients purposively sampled from the Victorian State Trauma Registry. We report one aspect here. Thematic analysis was undertaken of interview transcripts.

Results The importance of effective communication was a theme that had implications in the in-hospital, rehabilitation and community care settings. Effective communication occurred when service providers conveyed detailed information in a sensitive and clear manner, frequently and actively involved patients in discussion, and were responsive to patients' questions. Such interactions encouraged information exchange and shared decisionmaking. Ineffective communication arose predominately during in-hospital care and at discharge from inpatient facilities. Themes related to hospital care included limited contact with health professionals, inability to process information, indirect communication, and struggling to deal with multiple health professionals. At hospital and rehabilitation discharge, themes included insufficient patient engagement, inadequate information flow and feeling disregarded. Ineffective communication resulted in discontinuity of care and preventable health problems.

Conclusions The communication and information needs of seriously injured patients were inconsistently met over the course of their recovery. The findings reveal the need for trauma care systems to support relevant training of service providers, engage patients in planning decisions, and provide information in appropriate forms. 